\title{
On rectangular Kronecker coefficients
}

\author{
Laurent Manivel
}

Received: 20 July 2009 / Accepted: 3 June 2010 / Published online: 29 June 2010

(C) Springer Science+Business Media, LLC 2010

\begin{abstract}
We show that rectangular Kronecker coefficients stabilize when the lengths of the sides of the rectangle grow, and we give an explicit formula for the limit values in terms of invariants of $\mathfrak{s} l_{n}$.
\end{abstract}

Keywords Kronecker coefficient $\cdot$ Invariant theory $\cdot$ Symmetric group $\cdot$ Special linear group · Tensor product

\section{Introduction}

Kronecker coefficients are structure constants for tensor products of irreducible complex representations of symmetric groups. More precisely, the irreducible representations of $\mathcal{S}_{n}$, the group of permutations of $n$ symbols, are indexed by partitions of $n$, see $[8$, I.7]. Denote by $[\lambda]$ the representation associated to a partition $\lambda$. Then we can decompose

$$
[\lambda] \otimes[\mu]=\bigoplus_{\nu} k_{\lambda, \mu, \nu}[\nu],
$$

and it is a major unsolved problem to find a combinatorial rule for computing the Kronecker coefficients $k_{\lambda, \mu, \nu}$. Note that since [ $\nu$ ] is self-dual, $k_{\lambda, \mu, \nu}$ is symmetric with respect to $\lambda, \mu, v$.

Motivated by certain aspects of algebraic complexity theory (see [3] for an overview), we focus on a more specific problem. Suppose that $\mu=v=\left(d^{n}\right)$, the rectangle partition with $n$ parts equal to $d$. Can we compute the corresponding Kro-

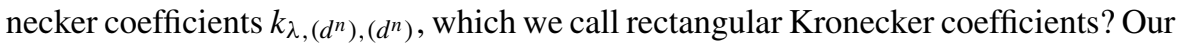
main result is the following: 
Theorem 1 Let $\lambda=(d n-|\rho|, \rho)$ for some partition $\rho$. Consider the Kronecker co-

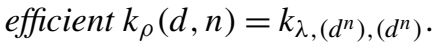

(1) $k_{\rho}(d, n)$ is a symmetric, non decreasing function of $n$ and $d$;

(2) for $d \geq|\rho|$, the Kronecker coefficient $k_{\rho}(d, n)$ is equal to

$$
k_{\rho}(n):=\operatorname{dim} S_{\rho}\left(\mathfrak{s l} l_{n}\right)^{\mathrm{GL}_{n}} .
$$

A few words of explanation are in order: the general complex linear group $\mathrm{GL}_{n}$ acts by conjugation on the Lie algebra $\mathfrak{s} l_{n}$ of traceless matrices. For any partition $\rho$ there is an induced action of $\mathrm{GL}_{n}$ on the Schur power $S_{\rho}\left(\mathfrak{s l} l_{n}\right)$, and $S_{\rho}\left(\mathfrak{s l} l_{n}\right)^{\mathrm{GL}_{n}}$ denotes the subspace of invariants of this action. (Note that we could replace $\mathrm{GL}_{n}$ by $\mathrm{SL}_{n}$.) Also, we have denoted by $|\rho|$ the sum of the parts of the partition $\rho$. In the sequel we will denote by $\ell(\rho)$ its length, defined as the number of non-zero parts.

\section{Proof of the theorem}

We first use Schur-Weyl duality to translate the computations of Kronecker coefficients to the setting of $\mathrm{GL}_{m}$-modules and their Schur powers, or equivalently, to a problem involving symmetric functions. By Schur-Weyl duality, we mean the following statement (see, e.g., [6, Theorem 2.4.3]). For a complex vector space $V$, the $\mathcal{S}_{n} \times \mathrm{GL}(V)$-module $V^{\otimes n}$ decomposes as

$$
V^{\otimes n}=\bigoplus_{\substack{|\lambda|=n, \ell(\lambda) \leq \operatorname{dim} V}}[\lambda] \otimes S_{\lambda} V .
$$

An immediate consequence of this statement, which can serve as a definition of Schur functors, is that

$$
S_{\lambda} V=\operatorname{Hom}_{\mathcal{S}_{n}}\left([\lambda], V^{\otimes n}\right),
$$

where by $\operatorname{Hom}_{\mathcal{S}_{n}}$ we mean the space of $\mathcal{S}_{n}$-equivariant homomorphisms. The statement of Schur-Weyl duality can then be translated into the fact that this defines an irreducible GL( $V)$-module, with highest weight $\lambda$. Another straightforward consequence is that for two vector spaces $U, V$ and three partitions $\lambda, \mu, v$ of the same integer $n$, the multiplicity of $S_{\mu} U \otimes S_{\nu} V$ inside $S_{\lambda}(U \otimes V)$ is equal to the Kronecker coefficient $k_{\lambda, \mu, v}$ (at least for $\ell(\mu) \leq \operatorname{dim} U, \ell(\nu) \leq \operatorname{dim} V$ and $\ell(\lambda) \leq \operatorname{dim} U \operatorname{dim} V$ ).

Remark 1 If $u, v$ are the dimensions of $U$, then $S_{\mu+\left(a^{u}\right)} U=S_{\mu} U \otimes(\operatorname{det} U)^{a}$. Since $\operatorname{det}(U \otimes V)=\operatorname{det}(U)^{v} \otimes \operatorname{det}(V)^{u}$, the identity $S_{\lambda+\left(a^{u v}\right)}(U \otimes V)=S_{\lambda}(U \otimes V) \otimes$ $\operatorname{det}(U \otimes V)^{a}$ implies that

$$
k_{\lambda+\left(a^{u v}\right), \mu+\left((a v)^{u}\right), v+\left((a u)^{v}\right)}=k_{\lambda, \mu, v}
$$

for any $\lambda, \mu, v$ such that $\ell(\mu) \leq u, \ell(v) \leq v, \ell(\lambda) \leq u v$. This is the main result of [11]. 
Suppose now that $V=W \oplus L$ for some vector spaces $W, L$, with $\operatorname{dim} L=1$. We compute $S_{\lambda}(U \otimes V)$ in two different ways. First, we can use the previous remark and write

$$
S_{\lambda}(U \otimes V)=\bigoplus_{\mu, \nu} k_{\lambda, \mu, \nu} S_{\mu} U \otimes S_{\nu}(W \oplus L)=\bigoplus_{\substack{\mu, \nu, \theta \\ \nu \mapsto \theta}} k_{\lambda, \mu, \nu} S_{\mu} U \otimes S_{\theta} W \otimes L^{|\nu|-|\theta|}
$$

Here we have used a version of the Pieri formula,

$$
S_{\nu}(W \oplus L)=\bigoplus_{\nu \mapsto \theta} S_{\theta} W \otimes L^{|v|-|\theta|},
$$

where the notation $v \mapsto \theta$ means that $v_{i} \geq \theta_{i} \geq v_{i+1}$ for all $i$. This formula is a special case of a more general formula which is also a consequence of Schur-Weyl duality: applying a Schur functor to a direct sum, one gets

$$
S_{\lambda}(E \oplus F)=\bigoplus_{|\alpha|+|\beta|=|\lambda|} c_{\lambda}^{\alpha, \beta} S_{\alpha} E \otimes S_{\beta} F
$$

where the Littlewood-Richardson coefficient $c_{\lambda}^{\alpha, \beta}$ can be defined as the multiplicity of $S_{\lambda} G$ inside the tensor product $S_{\alpha} G \otimes S_{\beta} G$, for $G$ of large enough dimension. We can apply this formula to derive

$$
S_{\lambda}(U \otimes V)=S_{\lambda}(U \otimes W \oplus U \otimes L)=\bigoplus_{|\alpha|+|\beta|=|\lambda|} c_{\lambda}^{\alpha, \beta} S_{\alpha}(U \otimes W) \otimes S_{\beta} U \otimes L^{|\beta|}
$$

and then, decomposing $S_{\alpha}(U \otimes W)$ with the help of Kronecker coefficients,

$$
S_{\lambda}(U \otimes V)=\bigoplus_{\substack{\alpha, \beta, \rho, \theta \\|\alpha|+|\beta|=|\lambda|}} c_{\lambda}^{\alpha, \beta} k_{\alpha, \rho, \theta} S_{\rho} U \otimes S_{\beta} U \otimes S_{\theta} W \otimes L^{|\beta|} .
$$

Comparing with (1), we get the identity

$$
\sum_{\nu \mapsto \theta} k_{\lambda, \mu, \nu}=\sum_{\alpha, \beta, \rho} c_{\lambda}^{\alpha, \beta} k_{\alpha, \rho, \theta} c_{\mu}^{\rho, \beta} .
$$

Now we specialize to the case where $\lambda=\mu=\left(d^{n}\right)$. We get

$$
\sum_{\nu \mapsto \theta} k_{\left(d^{n}\right),\left(d^{n}\right), \nu}=\sum_{\alpha, \beta, \rho} c_{\left(d^{n}\right)}^{\alpha, \beta} k_{\alpha, \rho, \theta} c_{\left(d^{n}\right)}^{\rho, \beta} .
$$

The Littlewood-Richardson coefficients in this formula are easy to understand: we have the following folklore lemma, which is an easy application of the LittlewoodRichardson rule (and also a version of Poincaré duality for Grassmannians).

Lemma 1 If the Littlewood-Richardson $c_{\left(d^{n}\right)}^{\alpha, \beta}$ is non-zero, then $\alpha$ and $\beta$ are complementary partitions in the rectangle $d \times n$; that is,

$$
\alpha_{i}+\beta_{n-i+1}=d \quad \forall i
$$


(In particular $\alpha_{1}, \beta_{1} \leq d$ and $\alpha_{n+1}=\beta_{n+1}=0$.) When this condition is satisfied, $c_{\left(d^{n}\right)}^{\alpha, \beta}=1$.

Proof Let $\beta^{c}$ be the complementary partition to $\beta$ in the rectangle $d \times n$. The Littlewood-Richardson rule asserts that $c_{\left(d^{n}\right)}^{\alpha, \beta}$ is the number of semi-standard skewtableaux $T$ of shape $\beta^{c}$ and weight $\alpha$, whose associated word $w(T)$ is a lattice permutation (see [8] for details). But the latter condition completely determines $T$ : for any $i$, the $i$-th line of $T$ must be filled with $i$ 's. And then its content is $\beta^{c}$.

This implies that in formula (3), the non-zero contributions to the right-hand side come from the partitions $\alpha, \beta, \rho$ contained in the rectangle $d \times n$ such that $\alpha=\rho$ is complementary to $\beta$. We can thus write:

$$
\sum_{\nu \mapsto \theta} k_{\left(d^{n}\right),\left(d^{n}\right), \nu}=\sum_{\alpha \subset d \times n} k_{\alpha, \alpha, \theta} .
$$

Observe that these two sums are of a quite different nature: the first one is taken over partitions $v$ of size $d n$; but the second one is taken over partitions $\alpha$ of the same size as $\theta$; and this size can be arbitrary. In particular, if we suppose that $|\alpha|=|\theta| \leq$ $\min (d, n)$, then the condition that $\alpha \subset d \times n$ is superfluous. If we simply ask that $|\theta| \leq d$, the condition $\alpha \subset d \times n$ amounts to the condition that the length of $\alpha$ does not exceed $n$, and we can rewrite the previous identity as

$$
\sum_{\nu \mapsto \theta} k_{\left(d^{n}\right),\left(d^{n}\right), \nu}=\sum_{\ell(\alpha) \leq n} k_{\alpha, \alpha, \theta} .
$$

In particular the right-hand side does not depend on $d$ if $d \geq|\theta|$.

Now, write $\nu=(d n-|\rho|, \rho)$ for some partition $\rho$, and observe that the condition that $v \mapsto \theta$ means that $d n-|\rho| \geq \theta_{1} \geq \rho_{1} \geq \theta_{2} \geq \rho_{2} \cdots$. Otherwise said, we ask that $d n-|\rho| \geq \theta_{1}$ and $\theta \mapsto \rho$. In particular, the latter condition implies that, $|\rho| \leq|\theta|$, and therefore the condition that $d n-|\rho| \geq \theta_{1}$ follows automatically as soon as $d \geq 2$. We can thus rewrite our identity as

$$
\sum_{\theta \mapsto \rho} k_{\left(d^{n}\right),\left(d^{n}\right),(d n-|\rho|, \rho)}=\sum_{\ell(\alpha) \leq n} k_{\alpha, \alpha, \theta} .
$$

In order to interpret the right-hand side, we apply the formula for Schur powers of tensor products to two vector spaces in duality. That is, we write

$$
S_{\theta}\left(V \otimes V^{\vee}\right)=\bigoplus_{\ell(\alpha), \ell(\beta) \leq \operatorname{dim} V} k_{\alpha, \beta, \theta} S_{\alpha} V \otimes S_{\beta} V^{\vee}
$$

By Schur's lemma, the space of GL(V)-invariants inside $S_{\alpha} V \otimes S_{\beta} V^{\vee}=$ $\operatorname{Hom}\left(S_{\beta} V, S_{\alpha} V\right)$ is non-zero if and only if $\alpha=\beta$, in which case it is one-dimensional. Letting $V=\mathbb{C}^{n}$, we deduce that

$$
\operatorname{dim} S_{\theta}\left(\operatorname{End}_{n}\right)^{\mathrm{GL}_{n}}=\sum_{\ell(\alpha) \leq n} k_{\alpha, \alpha, \theta} \cdot
$$


Here, $\operatorname{End}_{n}=\mathfrak{s} l_{n} \oplus \mathbb{C}$, hence by the Pieri formula $S_{\theta}\left(\operatorname{End}_{n}\right)=\bigoplus_{\theta \mapsto \rho} S_{\rho}\left(\mathfrak{s l} l_{n}\right)$. We can thus rewrite the identity (5) between Kronecker coefficients as

$$
\sum_{\theta \mapsto \rho} k_{\left(d^{n}\right),\left(d^{n}\right),(d n-|\rho|, \rho)}=\sum_{\theta \mapsto \rho} \operatorname{dim} S_{\rho}\left(\mathfrak{s} l_{n}\right)^{\mathrm{GL}_{n}},
$$

for any $\theta$ such that $|\theta| \leq d$. But this implies that for any $\rho$ such that $|\rho| \leq d$,

$$
k_{\left(d^{n}\right),\left(d^{n}\right),(d n-|\rho|, \rho)}=\operatorname{dim} S_{\rho}\left(\mathfrak{s} l_{n}\right)^{\mathrm{GL}_{n}} .
$$

Indeed, $\theta \mapsto \rho$ implies $\theta=\rho$ or $|\rho|<|\theta|$, so by induction we are immediately done. This proves the second assertion of the Theorem.

The proof of the first assertion is straightforward. First, the sign representation $\left[1^{m}\right]$ of $\mathcal{S}_{m}$ has the property that its tensor product with an irreducible representation $[\lambda]$ is the irreducible representation $\left[\lambda^{\vee}\right]$, where $\lambda^{\vee}$ denotes the transpose partition to $\lambda$. Since $\left[1^{m}\right] \otimes\left[1^{m}\right]$ is just the trivial representation, this implies that $[\lambda] \otimes[\lambda]=$ $\left[\lambda^{\vee}\right] \otimes\left[\lambda^{\vee}\right]$. Applying this remark to a rectangular partition $\lambda=\left(d^{n}\right)$, we get $\lambda^{\vee}=$ $\left(n^{d}\right)$, hence

$$
k_{\rho}(d, n)=k_{\rho}(n, d) \quad \forall \rho .
$$

Second, this is a non decreasing function of $d$ because of the semi-group property of Kronecker coefficients: if $k_{\lambda, \mu, \nu}$ and $k_{\lambda^{\prime}, \mu^{\prime}, v^{\prime}}$ are non-zero, then $k_{\lambda+\lambda^{\prime}, \mu+\mu^{\prime}, v+v^{\prime}}$ is also non-zero, and more precisely,

$$
k_{\lambda+\lambda^{\prime}, \mu+\mu^{\prime}, \nu+v^{\prime}} \geq \max \left(k_{\lambda, \mu, \nu}, k_{\lambda^{\prime}, \mu^{\prime}, v^{\prime}}\right) .
$$

Indeed, let $E, F, G$ be three vector spaces and $U$ be the unipotent subgroup of $\mathrm{GL}(E) \times \mathrm{GL}(F) \times \mathrm{GL}(G)$ consisting of triples of strictly triangular matrices, with respect to some choice of basis. Let $T$ be the maximal torus consisting of triples of diagonal matrices in the same basis. Then it is another consequence of Schur-Weyl duality that the invariant algebra $A=\operatorname{Sym}(E \otimes F \otimes G)^{U}$, which is a $T$-module, has weight spaces $A_{\lambda, \mu, \nu}$ of dimension $k_{\lambda, \mu, \nu}$. Then the semi-group property follows from the obvious fact that $A$ is an algebra without zero-divisors.

Since $k_{1^{n}, 1^{n}, n}$ is equal to one, we are done.

Remark 2 In the second assertion of the Theorem, we can improve the bound on $d$ as follows:

$$
k_{\rho}(d, n)=\operatorname{dim} S_{\rho}\left(\mathfrak{s} l_{n}\right)^{\mathrm{GL}_{n}}
$$

as soon as $2 d \geq|\rho|+\rho_{1}$. Indeed, for a Kronecker coefficient $k_{\alpha, \beta, \theta}$ to be non-zero, we need that $m-\theta_{1} \leq\left(m-\alpha_{1}\right)+\left(m-\beta_{1}\right)$, where $m=|\alpha|=|\beta|=|\theta|[7$, Theorem 2.9.22]. Therefore $k_{\alpha, \alpha, \theta} \neq 0$ implies that $2 \lambda_{1} \leq|\theta|+\theta_{1}$, so that in (4) we can replace the condition that $\alpha \subset d \times n$ by $\ell(\alpha) \leq n$ as soon as $2 d \geq|\theta|+\theta_{1}$.

Remark 3 The case where $n=2$ has been considered in [9], where we proved by completely different methods that $k_{\rho}(d, 2)$ does not depend on $d$. More precisely, this Kronecker coefficient is equal to one if and only if $\rho$ is even of length at most 
three or odd of length three (by an odd partition we mean a partition all of whose parts are odd), and zero otherwise. This result was also obtained independently in [5]. From the Theorem we deduce the corresponding statement for the dimension of $S_{\rho}\left(\mathfrak{s} l_{2}\right)^{\mathrm{GL}_{2}}$.

\section{Complements}

\subsection{Invariants of matrices}

We have met the identity

$$
\operatorname{dim} S_{\theta}\left(\operatorname{End}_{n}\right)^{\mathrm{GL}_{n}}=\sum_{\ell(\alpha) \leq n} k_{\alpha, \alpha, \theta} .
$$

For a given $\theta$, it implies that the dimension of $S_{\theta}\left(\mathrm{End}_{n}\right)^{\mathrm{GL}_{n}}$ is a non decreasing function of $n$, which is equal for $n \geq|\theta|=m$ to the multiplicity of $[\theta]$ inside

$$
C_{m}=\bigoplus_{|\alpha|=m}[\alpha] \otimes[\alpha] .
$$

Observe that $C_{m}$ is nothing other that the conjugating representation of $\mathcal{S}_{m}$ over $\mathbb{C}\left[\mathcal{S}_{m}\right]$. Indeed, the regular representation of $\mathcal{S}_{m}$ over $\mathbb{C}\left[\mathcal{S}_{m}\right]$, with its $\mathcal{S}_{m} \times \mathcal{S}_{m}$ module structure defined by the left and right actions of $\mathcal{S}_{m}$, is given by the same formula (but with exterior tensor products), and the conjugating representation is just the regular representation restricted to the diagonal embedding of $\mathcal{S}_{m}$ inside $\mathcal{S}_{m} \times \mathcal{S}_{m}$. (Of course this is not specific to symmetric groups, see e.g. [12, Theorem 3.2.2].) We denote $C_{m}=\operatorname{diag} \mathbb{C}\left[\mathcal{S}_{m}\right]$.

A more transparent way to understand the previous identity is to observe that it is closely related to the fundamental theorems for invariants of matrices. Indeed (see, e.g., [10, Chap. 11, §8], or [4]), the first fundamental theorem asserts that the $\mathcal{S}_{m}$ equivariant map

$$
f_{m, n}: \operatorname{diag} \mathbb{C}\left[\mathcal{S}_{m}\right] \longrightarrow\left(\operatorname{End}_{n}^{\otimes m}\right)^{\mathrm{GL}_{n}}
$$

mapping a permutation $\sigma$ to $f_{m, n}(\sigma) \in \operatorname{End}_{n}^{\otimes m}=\operatorname{End}\left(\mathbb{C}^{n}\right)^{\otimes m}$, defined by

$$
f_{m, n}(\sigma)\left(X_{1} \otimes \cdots \otimes X_{m}\right)=X_{\sigma(1)} \otimes \cdots \otimes X_{\sigma(m)},
$$

is surjective. Moreover, the second fundamental theorem asserts that the kernel of $f_{m, n}$ is the sum of the submodules $[\alpha] \otimes[\alpha]$ of $\operatorname{diag} \mathbb{C}\left[\mathcal{S}_{m}\right]$, for $\ell(\alpha)>n$. In particular, $f_{m, n}$ is an isomorphism for $n \geq m$.

\subsection{Derangements}

How can we pass from $\operatorname{End}_{n}^{\otimes m}$ to $\mathfrak{s} l_{n}^{\otimes m}$ ? A first observation is that, since $\operatorname{End}_{n}=$ $\mathbb{C} I_{n} \oplus \mathfrak{s l} l_{n}$, and the identity $I_{n}$ is certainly $\mathrm{GL}_{n}$-invariant, we have

$$
\left(\mathrm{End}_{n}^{\otimes m}\right)^{\mathrm{GL}_{n}} \simeq \bigoplus_{p=0}^{m}\left(\begin{array}{c}
m \\
p
\end{array}\right)\left(\mathfrak{s l} l_{n}^{\otimes p}\right)^{\mathrm{GL}_{n}} .
$$


Since the left-hand side, for $n \geq m$, has dimension $m$ !, we easily deduce that

$$
\operatorname{dim}\left(\mathfrak{s l} l_{n}^{\otimes m}\right)^{\mathrm{GL}_{n}}=\sum_{p=0}^{m}(-1)^{p}\left(\begin{array}{c}
m \\
p
\end{array}\right)(m-p) !=D_{m},
$$

the number of derangements, that is, fixed-point free permutations in $\mathcal{S}_{m}$. This was already observed in [1], but only under the weaker condition that $n \geq 2 m$.

We can be more precise by making the following observation. We have

$$
\left(\mathfrak{s} l_{n}^{\otimes m}\right)^{\mathrm{GL}_{n}}=\left(\operatorname{End}_{n}^{\otimes m}\right)^{\mathrm{GL}_{n}} \cap \mathfrak{s} l_{n}^{\otimes m},
$$

and the subspace $\mathfrak{s} l_{n}^{\otimes m}$ of $\operatorname{End}_{n}^{\otimes m}$ can be characterized as the intersection of the kernels of the contractions

$$
c_{k}: \operatorname{End}_{n}^{\otimes m} \rightarrow \operatorname{End}_{n}^{\otimes m-1}
$$

defined, for $1 \leq k \leq m$, by applying the trace morphism to the $k$ th factor of $\operatorname{End}_{n}^{\otimes m}$.

Consider an element $f_{n, m}(\sigma)$ for some $\sigma \in \mathcal{S}_{m}$. Once we have chosen a basis $e_{1}, \ldots, e_{n}$ of $\mathbb{C}^{n}$, we can write it explicitly as

$$
f_{n, m}(\sigma)=\sum_{i_{1}, \ldots, i_{m}=1}^{n}\left(e_{i_{1}}^{\vee} \otimes e_{i_{\sigma(1)}}\right) \otimes \cdots \otimes\left(e_{i_{m}}^{\vee} \otimes e_{i_{\sigma(m)}}\right) .
$$

An easy computation then shows that a linear combination $a=\sum_{\sigma} a_{\sigma} \sigma \in \mathbb{C}\left[\mathcal{S}_{m}\right]$ belongs to the kernel of $c_{m}$, the last contraction, if and only if

$$
n a_{(\tau, m)}+\sum_{k=1}^{m-1} a_{s_{k, m}(\tau, m)}=0
$$

for all $\tau \in \mathcal{S}_{m-1}$. Here we have denoted by $(\tau, m) \in \mathcal{S}_{m}$ the permutation deduced from $\tau$ by adding the fixed point $m$, and by $s_{k, m}$ the transposition exchanging $k$ and $m$. In this identity, note that $(\tau, m)$ has one more fixed point than $\tau$, while $s_{k, m}(\tau, m)$ has either the same number of fixed points as $\tau$, or one less. This implies, inductively, that if $a=\sum_{\sigma} a_{\sigma} \sigma$ is annihilated by the contractions $c_{1}, \ldots, c_{m}$, then all the coefficients of $a$ can be expressed in terms of the coefficients $a_{\sigma}$, for $\sigma$ describing the set of fixed-point free permutations-or derangements. Since we know that the dimension of the space of such $a$ 's is precisely the set of derangements, the coefficients of the derangements must be independent. Let diag $\mathbb{C}\left[\mathcal{S}_{m}\right]_{\text {fpf }} \subset \operatorname{diag} \mathbb{C}\left[\mathcal{S}_{m}\right]$ be the submodule defined by the derangements in $\mathcal{S}_{m}$. We deduce from the preceding discussion:

Proposition 1 For $n \geq m$, there is an isomorphism of $\mathcal{S}_{m}$-modules

$$
\operatorname{diag} \mathbb{C}\left[\mathcal{S}_{m}\right]_{\mathrm{fpf}} \simeq\left(\mathfrak{s} l_{n}^{\otimes m}\right)^{\mathrm{GL}_{n}}
$$

This has the following consequence for rectangular Kronecker coefficients. 
Corollary 1 The limit value $k_{\rho}$ of $k_{\rho}(d, n)$ for $d, n \geq|\rho|$, is equal to the multiplicity of $[\rho]$ inside $\operatorname{diag} \mathbb{C}\left[\mathcal{S}_{m}\right]_{\text {fpf }}$, the fixed-point free part of the conjugating representation.

Although $D_{m}$ grows very fast with $m$, the coefficients $k_{\rho}$ seem to remain much smaller. For $m \leq 6$ their values are given by the following table:

$$
\begin{aligned}
& m=2 \quad k_{2}=1, \quad k_{11}=0, \\
& m=3 \quad k_{3}=1, \quad k_{21}=0, \quad k_{111}=1, \\
& D_{2}=1 \text {, } \\
& m=4 \quad k_{4}=2, \quad k_{31}=0, \quad k_{22}=2, \quad k_{211}=1, \quad k_{14}=0, \\
& m=5 \quad k_{5}=2, \quad k_{41}=1, \quad k_{32}=2, \quad k_{311}=3, \quad k_{221}=1, \\
& k_{21^{3}}=1, \quad k_{15}=1, \quad D_{5}=44, \\
& m=6, \quad k_{6}=4, \quad k_{51}=1, \quad k_{42}=6, \quad k_{33}=1, \quad k_{411}=4, \\
& k_{321}=4, \quad k_{2^{3}}=5, \quad k_{31^{3}}=4, \quad k_{2211}=2, \quad k_{21^{4}}=2, \\
& k_{16}=0 \text {, } \\
& D_{3}=2 \text {, } \\
& D_{4}=9 \text {, } \\
& D_{6}=265 \text {. }
\end{aligned}
$$

\subsection{Symmetric and skew-symmetric invariants}

Certain submodules of the tensor algebra of $\mathfrak{s} l_{n}$ have a classical interpretation. Indeed, it is well known that $\mathrm{GL}_{n}$-invariants in the symmetric algebra $\operatorname{Sym}\left(\mathfrak{s} l_{n}\right)$ generate a polynomial algebra with generators in degree $2,3, \ldots, n$. On the other hand, $\mathrm{GL}_{n}$-invariants in the exterior algebra $\Lambda\left(\mathfrak{s} l_{n}\right)$ can be interpreted as invariant differential forms on the Lie group $\mathrm{SL}_{n}$, and it follows from classical results by Hopf and Samelson that $\Lambda\left(\mathfrak{s} l_{n}\right)^{\mathrm{GL}_{n}}$ is an exterior algebra with generators of odd degrees $3,5, \ldots, 2 n-1$. Rephrasing these facts, and taking Remark 2 into account, we get:

Corollary 2 (1) For $d \geq m$, the Kronecker coefficient $k_{(m)}(d, n)$ is equal to the number of partitions of $m$ into integers between 2 and $n$.

(2) For $d \geq \frac{m+1}{2}$, the Kronecker coefficient $k_{\left(1^{m}\right)}(d, n)$ is equal to the number of partitions of $m$ into distinct odd integers between 3 and $2 n-1$.

Note that partitions of $m$ into distinct odd integers are in correspondence with partitions $\lambda$ of $m$ equal to their own transpose. This is equivalent to the condition that $[\lambda] \otimes[\lambda]$ contains the sign representation, so that the number of partitions of $m$ into distinct odd integers can be expressed as

$$
p_{\text {odd }}^{0}(m)=\sum_{|\lambda|=m} k_{\lambda, \lambda,\left(1^{m}\right)} .
$$

\subsection{A birational map}

It may be possible to give a more transparent and more geometric proof of our results starting from the following observation. Let $E, F, G$ be three vector spaces of dimensions $n, n, g$, with $g \leq n^{2}$. Let $\gamma_{1}, \ldots, \gamma_{g}$ be a basis of $G$, and denote by $U_{G}$ the unipotent subgroup of $\mathrm{GL}(G)$ consisting of endomorphisms whose matrix in this basis is strictly upper-triangular. Consider a generic element $T \in E^{\vee} \otimes F \otimes G^{\vee}=$ $\operatorname{Hom}(G, \operatorname{Hom}(E, F))$. The image $T\left(\gamma_{1}\right)$ of $\gamma_{1}$ is an invertible morphism, which identifies $E$ with $F$. Under this identification, $T\left(\gamma_{1}\right)$ becomes the identity map, and $T$ can 
be interpreted as a tensor $\bar{T} \in \operatorname{Hom}\left(G /\left\langle\gamma_{1}\right\rangle, \operatorname{End}(E) /\left\langle\operatorname{Id}_{E}\right\rangle\right)$, where $\operatorname{End}(E) /\left\langle\operatorname{Id}_{E}\right\rangle$ can be identified with $\mathfrak{s l}(E)$. Since $T$ is generic and $g \leq n^{2}, \bar{T}$ is injective, and we deduce a flag $W_{1} \subset \cdots \subset W_{g-1} \subset \mathfrak{s l}(E)$, where $W_{k}$ has dimension $k$. Denoting by $\mathcal{F}_{g-1}(\mathfrak{s l}(E))$ the variety parameterizing such flags, this construction defines a rational map

$$
\pi: \mathbb{P}\left(E^{\vee} \otimes F \otimes G^{\vee}\right) / / \operatorname{SL}(E) \times \operatorname{SL}(F) \times U_{G} \longrightarrow \mathcal{F}_{g-1}(\mathfrak{s l}(E)) / / \operatorname{SL}(E)
$$

which is easily seen to be birational. The left-hand side is $\operatorname{Proj}(A)$, where

$$
A=\bigoplus_{\delta \geq 0} S^{\delta}\left(E^{\vee} \otimes F \otimes G^{\vee}\right)^{\mathrm{SL}(E) \times \mathrm{SL}(F) \times U_{G}}=\bigoplus_{d \geq 0} k_{\left(d^{n}\right),\left(d^{n}\right), \lambda}\left(S_{\lambda} G^{\vee}\right)^{U_{G}}
$$

is endowed with a natural action of the torus $T_{G}$ of diagonal matrices in GL( $\left.G\right)$, such that the one-dimensional vector space $\left(S_{\lambda} G^{\vee}\right)^{U_{G}}$ is acted on by the character defined by $\lambda$. In consequence, $A$ is graded over the character group of $T_{G}$, and the dimension

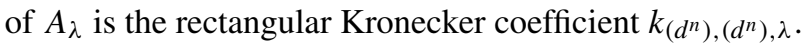

On the right-hand side, consider a line bundle $\mathcal{L}_{\alpha}$, whose fiber over a point of $\mathcal{F}_{g-1}(\mathfrak{s l}(E))$ defined by a flag $W_{1} \subset \cdots \subset W_{g-1} \subset \mathfrak{s l}(E)$ is $W_{1}^{\alpha_{1}} \otimes\left(W_{2} / W_{1}\right)^{\alpha_{2}} \otimes$ $\cdots \otimes\left(W_{g-1} / W_{g-2}\right)^{\alpha_{g-1}}$. If $\alpha$ is a partition, then by the Borel-Weil theorem

$$
H^{0}\left(\mathcal{F}_{g-1}(\mathfrak{s l}(E)), \mathcal{L}_{\alpha}^{-1}\right) \simeq S_{\alpha} \mathfrak{s l}(E)
$$

Moreover, any $\operatorname{SL}(E)$-invariant section of $\mathcal{L}_{\alpha}^{-1}$ defines, through $\pi$, a rational function on $\mathbb{P}\left(E^{\vee} \otimes F \otimes G^{\vee}\right) / / \operatorname{SL}(E) \times \operatorname{SL}(F) \times U_{G}$. Multiplying this function by a $d$-th power of $\gamma_{1}^{n} \in S^{n} G \simeq \Lambda^{n} E \otimes \Lambda^{n} F^{\vee} \otimes S^{n} G \subset S^{n}\left(E \otimes F^{\vee} \otimes G\right)$, this rational function will become regular for $d$ large enough (this is because in the construction of $\pi$ we have inverted $T\left(\gamma_{1}\right)$, and we are now multiplying by some power of its determinant). The weight of the resulting section with respect to $T_{G}$ is easily computed to be (nd $-|\alpha|, \alpha)$, and we finally get a map

$$
S_{\alpha} \mathfrak{s l}(E)^{\mathrm{SL}(E)} \longrightarrow A_{(n d-|\alpha|, \alpha)} .
$$

Assertion (2) of Theorem 1 can thus be translated into the claim that, for $d$ large enough, this map is an isomorphism.

\subsection{A question}

We have seen that the semi-group property of Kronecker coefficients, and the obvious fact that $k_{\left(1^{n}\right),\left(1^{n}\right),(n)}=1$, is enough to ensure that the Kronecker coefficient we denoted $k_{\rho}(d, n)$ is a non-decreasing function of $n$ and $d$. More generally, for any integer $\delta$ and any partition $\lambda$ such that $k_{\left(\delta^{n}\right),\left(\delta^{n}\right), \lambda} \neq 0$, we can conclude that for any integer $d$ and any partition $\mu$ the Kronecker coefficient $k_{(d+m \delta)^{n},(d+m \delta)^{n}, \mu+m \lambda}$ is a non-decreasing function of $m$. How does it grow with $m$ ? Can one give an explicit equivalent when $m \rightarrow+\infty$ ? When is there a finite limit?

Of course this problem can also be considered for general Kronecker coefficients. The limit value of $k_{m+\alpha, m+\beta, m+\gamma}$, for $m \rightarrow+\infty$, has been computed in [2, 3.4]. 


\section{References}

1. Benkart, G., Doty, S.: Derangements and tensor powers of adjoint modules for $\mathfrak{s} l_{n}$. J. Algebr. Comb. 16(1), 31-42 (2002)

2. Brion, M.: Stable properties of plethysm: on two conjectures of Foulkes. Manuscr. Math. 80(4), 347371 (1993)

3. Buergisser, P., Landsberg, J.M., Manivel, L., Weyman, J.: An overview of mathematical issues arising in the geometric complexity theory approach to VP vs VNP. arXiv:0907.2850

4. Formanek, E.: The invariants of $n \times n$ matrices. In: Invariant Theory. Lecture Notes in Math., vol. 1278, pp. 18-43. Springer, Berlin (1987)

5. Garsia, A., Wallach, N., Xin, G., Zabrocki, M.: Kronecker coefficients via symmetric functions and constant term identities. Preprint (2008)

6. Howe, R.: Perspectives on invariant theory: Schur duality, multiplicity-free actions and beyond. In: The Schur Lectures (1992) (Tel Aviv). Israel Math. Conf. Proc., vol. 8, pp. 1-182. Bar-Ilan Univ., Ramat Gan (1995)

7. James, G., Kerber, A.: The Representation Theory of the Symmetric Group. Encyclopedia of Mathematics and its Applications, vol. 16. Addison-Wesley, Reading (1981)

8. Macdonald, I.G.: Symmetric Functions and Hall Polynomials, 2nd edn. Oxford University Press, Oxford (1995)

9. Manivel, L.: A note on certain Kronecker coefficients. Proc. Am. Math. Soc. 138, 1-7 (2010). arXiv: 0809.3710

10. Procesi, C.: Lie Groups, An Approach Through Invariants and Representations. Universitext. Springer, Berlin (2007)

11. Vallejo, E.: A stability property for coefficients in Kronecker products of complex $S_{n}$ characters. Electron. J. Comb. 16(1) (2009), Note 22, 8 pp.

12. Weintraub, S.: Representation Theory of Finite Groups: Algebra and Arithmetic. Graduate Studies in Mathematics, vol. 59. AMS, Providence (2003) 